\title{
Gamma-rays and Neutrinos from Hadronic Interactions in AGN Emission Models
}

\section{A. Reimer*}

Leopold-Franzens-Universität Innsbruck, Institut für Theoretische Physik, und Institut für Astround Teilchenphysik, 6020, Innsbruck, Austria

E-mail: anita.reimer@uibk.ac.at

The material of relativistic jets of active galactic nuclei is composed of leptons and hadrons. If a significant fraction of the overall jet power is converted into energizing protons to relativistic energies, along with the leptons, hadronic interactions play a role in converting particle energy into radiation and neutrino production. I will discuss the physics of hadronic blazar emission models with focus on photon and neutrino production, and compare in the gamma-ray range with the foreseen capabilities of the Cherenkov Telescope Array (CTA).

AGN Physics in the CTA Era-AGN2011,

May 16-17, 2011

Toulouse, France

\footnotetext{
*Speaker.

${ }^{\dagger}$ AR acknowledges support by Marie Curie IRG grant 248037 within the FP7 Program.
} 


\section{Introduction}

The census of GeV-detected blazars has dramatically increased from less than 100 blazars as detected by the EGRET instrument onboard the Compton Gamma-Ray Observatory to nearly $10^{3}$ Fermi-LAT (=Large Area Telescope)-detected high-latitude objects that have been associated with AGN after two years of observations [3]. The (nearly permanent) survey observation mode of the LAT-instrument onboard Fermi has triggered many follow-up observations of selected AGN also with the permanently improving instrument capabilities of modern Cherenkov-telescopes (ACTs). As a result, blazars of all subtypes (FSRQs, all types of BL Lac objects: low-frequency peaked BL Lacs (LBLs), intermediate-frequency peaked BL Lacs (IBLs), high-frequency-peaked BL Lacs (HBLs)) have meanwhile been detected at VHEs, thereby nearly doubling the census of blazars detected in this extreme photon energy band during the last two years.

Among the most striking properties of blazars is their variability at all wavelengths with variability time scales from weeks to months in the radio domain, down to even minutes in a few cases at VHEs (e.g., PKS 2155-304 [6], Mkn 501 [8]. Such extreme behaviour occurs in general during an enhanced flux and variability stage. The greatly improved sensitivity of gamma-ray instruments, however, allows meanwhile to detect a number of blazars at various variability stages including a quiescent non-variable state (see e.g., [2] for a study of gamma-ray variability properties of blazars).

The SED of jetted AGN consists of two broad components. The low-energy component is in general attributed to synchrotron radiation from relativistic electrons $\left(\mathrm{e}^{-}\right)$and positrons $\left(\mathrm{e}^{+}\right)$in a relativistically moving "blob" in the jet. Despite the rapid increase of photon data in recent years the origin of the high-energy hump is still a matter of debate, depending strongly on the overall jet composition.

Accordingly, two types of emission models have emerged during the last decade. So-called "leptonic" models consider relativistic $\mathrm{e}^{-} \mathrm{e}^{+}$as the emitting relativistic particle population, while in so-called "hadronic" emission models the relativistic jet material is composed of relativistic protons (p) and $e^{-}$. In both types of jet material cold pairs and/or protons may exist as well, allowing charge neutrality to be fulfilled. So-called "lepto-hadronic emission models" follow the same physics as "hadronic emission models". In the following I will therefore follow the historical path and use the term "hadronic model" for describing radiation from relativistic $\mathrm{e}^{-} \mathrm{p}$ jet matter compositions.

The historical motivation to consider relativistic protons in AGN jets is linked with the observation of ultra-high energy cosmic rays (UHECRs) up to several tens of EeV impinching the Earth from all directions. Indeed, AGN jets are among the prime candidate sources for these extremely energetic events. They fulfill all required criteria (e.g., [15]): Luminous AGN jets are located within the GZK horizon $\left(\sim 10^{2} \mathrm{Mpc}\right)$, provide sufficient power to potentially accelerate the charged jet particles beyond EeV scales and contribute significantly to the overall observed cosmic ray energetics, and fulfill the so-called "Hillas criterium" [17].

Observational indications of the existence of protons in AGN jets are meanwhile accumulating from various directions: The non-detection of the predicted soft X-ray excess radiation and X-ray precursors to blazar flares from bulk Comptonization of cold pairs in quasars with strong accretion disk radiation puts serious limits to the jet's overall pair content (e.g., [34]). The modeling of blazar SEDs with leptonic models reveals a total luminosity in pairs and magnetic field that is significantly 
less than the observed radiative power $L_{r a d}$ in the comoving frame, $L_{r a d} / \Gamma^{2}$ (e.g., [13]), with $\Gamma$ the bulk Lorentz factor. Energy conservation thus requires the existence of nucleons and/or nuclei in such blazar jets, a robust lower limit of the total jet power is given by $L_{j e t}>L_{\text {rad }} / \Gamma^{2}$. The role of pairs as jet energy carrier is therefore negligible, making hadrons the energetically and dynamically dominating matter component in blazar jets. What remains unclear so far are the properties of this hadron component, namely if there are relativistic protons, their spectrum and energy density.

\section{Photon and particle production in hadronic blazar emission models}

In hadronic jet emission models, both a relativistic electron and proton population exist in the emitting magnetized ${ }^{1}$ "blob" which itself moves with relativistic speed $\beta c$ along the jet axis. As a consequence, leptonic and hadronic ${ }^{2}$ processes must be fully considered: synchrotron radiation and inverse Compton scattering of charged particles, photon-photon and Bethe-Heitler pair production, photomeson production, inelastic nucleon-nucleon interactions, neutron decay. In this sense, leptonic emission models constitute a part of hadronic emission models.

Comparing electron and proton energy loss rates on finds that the photon production rate from electron interactions is in general larger than from proton interactions, electrons loose their energy more rapidly in dense radiative environments than protons. This makes electrons efficient radiators, nucleons - irrespective of whether they are cold or relativistic - are excellent energy carriers. Both properties are required in AGN jets.

In the following I will focus on the hadronic emission models where particle-photon interactions dominate over particle-particle interactions (e.g., [19, 26, 10, 22, 24, 25, 9]). Because the interaction threshold for Bethe-Heitler pair production is located at lower center-of-momentum frame (CMF) energies than for photomeson production, energy loss rates below the pion photoproduction threshold are dominated by pair production. Above this latter threshold energy losses from photomeson production typically dominate over those from pair production due to their several orders of magnitude greater inelasticity, ranging between $\sim 0.2$ in steep power-law target photon fields to nearly $\sim 0.5$ in very flat target photon fields [31, 32]. Photomeson production also allows proton to neutron conversion (and vice versa) via charge exchange (e.g. [23]) ${ }^{3}$. As a consequence collimated neutron beams may form $[16,9]$ which can be used to transport a significant portion of the initial energy to large distances from the black hole.

There are various target photon fields for particle-photon interactions in AGN jets: the internal jet synchrotron photon field $[19,24,25]$, and fields external to the jet such as direct accretion disk radiation [26, 10], jet or accretion disk radiation reprocessed in the BLR [9], or radiation by the dusty torus. The secondary (stable) particles from those are $\gamma$-rays, pairs and neutrinos $(v)$. For

\footnotetext{
${ }^{1}$ In the following I consider the magnetic field uniformly and isotropically distributed in the comoving "blob" frame for simplicity. Other scenarios may consider stochastic magnetic fields (e.g., [20] in the case of GRBs) giving rise to jitter radiation in the relativistic regime.

${ }^{2}$ The following restricts to proton interactions only. Heavy nuclei are generally more likely to photo-disintegrate into protons and neutrons in dense radiation fields rather than suffer photomeson production losses. Photo-disintegration has, however, recently been shown to fail providing an efficient process for VHE photon production [7], leaving protons as a more efficient photon producer.

${ }^{3}$ e.g., in hadronic $\mathrm{p} \gamma$-interactions $30 \%-70 \%$ of the initial protons are converted into neutrons [23]
} 
power-law target photon fields the resulting $\gamma$-ray to $v$ energy content $E_{\gamma} / E_{v}{ }^{4}$ lies around unity $(\sim 1-1.4)$ for a wide spectral index range, with $10 \%$ (8\%) for steep power-laws to $25 \%$ (20\%) in case of flat power-laws of the initial proton energy stored into photons (neutrinos) (see Figure 1; [31]).

Comparing the energy loss time scales from synchrotron radiation of muons in typically proposed blazar magnetic field strengths ${ }^{5}$ with their decay time scales one finds that for sufficient energetic muons, such as e.g., produced in photomeson production initiated by UHE protons, muon synchrotron losses prior to their decay can not be neglected (e.g., [28]). This applies similarly also to charged pions and kaons (e.g., [25]). Indeed, if field strengths are sufficiently high ( $\geq$ several tens of Gauss) p-[22, 4] $\mu^{ \pm}$- and $\pi^{ \pm}$-synchrotron radiation may constitute an important high energy photon component.

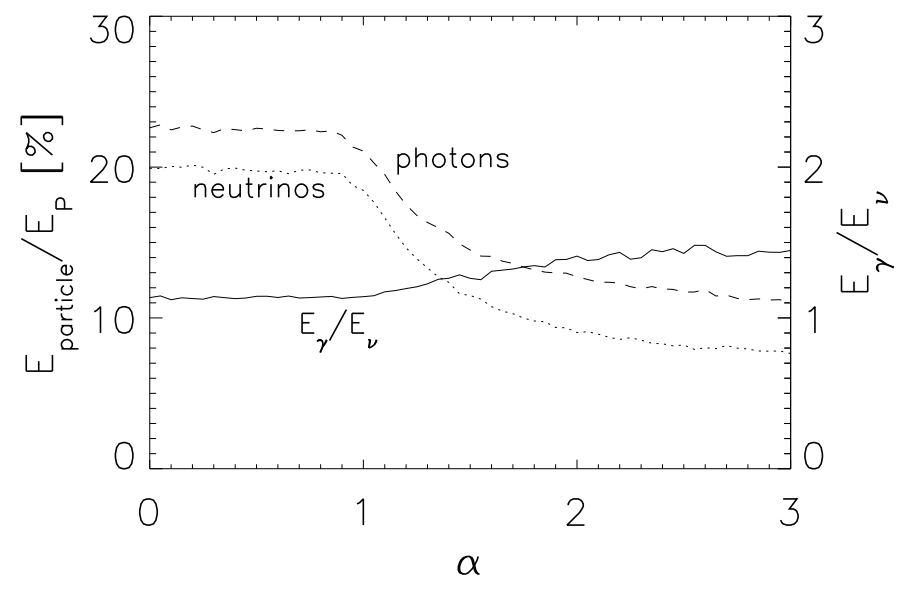

Figure 1: Photon and neutrino energy content per primary nucleon energy in photomeson production in power-law target photon fields with photon index $\alpha$ [31]. $e^{ \pm}$are assumed to be $100 \%$ radiatively efficient.

Very high energy photons (produced, e.g., through decay of photomeson produced $\pi \mathrm{s}$ ) may find the emission region optically thick: a pair cascade then develops which redistributes the power from very high to lower energies. Depending on the overall field to photon energy density in the emission region synchrotron- [19, 24, 25, 9] or inverse Compton supported pair cascades [9] may dominate. Electromagnetic cascades can be initiated by photons from $\pi^{0}$-decay (" $\pi^{0}$-cascade"), electrons from the $\pi^{ \pm} \rightarrow \mu^{ \pm} \rightarrow e^{ \pm}$decay (" $\pi^{ \pm}$-cascade"), $p$-synchrotron photons (" $p$-synchrotron cascade"), and $\mu$-, $\pi$ - and $K$-synchrotron photons (" $\mu^{ \pm}$-synchrotron cascade"). Ref. [24] and ref. [25] have shown that the " $\pi^{0}$ cascades" and " $\pi^{ \pm}$cascades" generate rather featureless $\gamma$-ray spectra, in comparison to " $p$-synchrotron cascades" and " $\mu^{ \pm}$-synchrotron cascades" which produce distinct features in the high energy SED.

Generally, hadronic AGN emission models also consider all leptonic processes (including inverse Compton scattering off low energy photons) owing to the presence of ultrarelativistic $\mathrm{e}^{-}$in the emission region. For sufficient high field strengths, any inverse Compton component is, however, strongly suppressed, leaving the proton-initiated radiation component as the dominating high energy emission mechanism.

\footnotetext{
${ }^{4} e^{ \pm}$are assumed to be $100 \%$ radiatively efficient.

5 except for one-zone SSC models where broadband SED modeling in general requires significantly lower field strengths
} 


\section{Efficiencies}

\subsection{Proton radiation efficiency}

The efficiency of producing photons from a proton of energy $\gamma_{p} m_{p} c^{2}$ within hadronic emission models is determined by the total photon production rate devided by the total proton loss rate. By averaging over the input proton spectrum $\propto \gamma_{p}^{-p}$ the total radiation efficience of protons is derived (e.g., [27]). Note that for large magnetic field strengths where parts of the neutrino producing channels are re-directed to photon producing channels via significant synchrotron losses of secondary charged particles produced in photomeson production (e.g., $\mu, \pi$, K, etc.). The fractional rate of photon production during one dynamical time scale after instantaneous injection of a power-law proton spectrum (here) $\propto E^{-2}$ into a power-law radiation field of given density can also be derived directly from a given blazar model taking into account all radiation processes self-consistently. Depending on the chosen parameter set of the model we find [33] the radiation efficiency of protons in hadronic emission models to range from percent (e.g., for parameter settings with an early cutoff of the proton spectrum, or very low magnetic field values) to tens of percent (e.g., for the case of a strong contribution from proton synchrotron radiation). The observed bolometric blazar luminosity can therefore be roughly estimated by $L_{\text {rad }} \sim \Gamma_{10} \xi_{p, 10} \xi_{\text {conv }, 10} L_{j e t}$ with $\Gamma$ the bulk Lorentz factor $\Gamma=10 \Gamma_{10}, \xi_{p}$ the proton radiation efficiency $\xi_{p}=0.1 \xi_{p, 10} \xi_{\text {conv }}$ the efficiency of converting jet power into relativistic particle energies $\xi_{c o n v}=0.1 \xi_{c o n v, 10}$, and $L_{j e t}$ the jet power. For a sufficient large jet power and favorable parameter choices typically observed blazar luminosities can therefore be accommodated in hadronic emission models.

\subsection{Neutrino Production Efficiency and Fluxes}

An inevitable by-product of hadronic interactions is the production of neutrinos. The neutrino production efficiency in hadronic emission models is calculated by analogy with the photon production efficiency. Because of proton losses such as, e.g. proton synchrotron radiation, competing with photomeson production and $\pi^{ \pm} / \mu^{ \pm}$-decay competing with synchrotron losses prior to its decay in highly magnetized environments it is clear that applying scaling relations to predict neutrino fluxes from gamma-ray observations can lead to neutrino flux upper limits only. We find [33] that in general the neutrino production efficiency is dependent on the model parameter values, reaching typically not more than $\sim 20 \%$. It can be extremely low in the case of high field strengths and weak target photon fields, and/or for low cutoff energies of the injected proton spectrum. Notably, in virtually no case is the neutrino production efficiency higher than the corresponding radiation efficiency.

Because of the potentially denser target photon field provided by powerful FSRQs as compared to BL Lacs, members of the former source class are considered a more promising neutrino source class as the close-by HBLs (e.g., [25]). The predicted diffuse neutrino fluxes are also not only highly model-dependent but also suffer from large uncertainties [25] which originate from unknowns in the model parameters, uncertainties of the class luminosity functions and evolution, etc. Current instruments are meanwhile capable of limiting the neutrino fluxes to not significantly above the "Waxman-Bahcall limit" (e.g., [1]) thereby ruling out hadronic neutrino production models with optimistically high predicted $v$-fluxes. 


\section{Hadronic blazar modeling along the sequence HBL - IBL - LBL - FSRQ}

There is overall consensus that synchrotron radiation from primary electrons dominates most parts of the low energy blazar SED in either model, leptonic or hadronic ${ }^{6}$. In the hadronic SynchrotronProton Blazar (SPB) model, e.g., the high energy bump stems mainly from direct proton, $\mu^{ \pm} / \pi^{ \pm}$ synchrotron radiation and their reprocessed components, and possibly contributions from the $\pi$ cascades [25]. Which of those processes dominate the SED at gamma-ray energies depends on the chosen parameter set. E.g., in environments with high field strengths but relatively weak low energy photon fields a dominance of proton synchrotron radiation is expected with a negligible cascading component. Such environments offer, e.g., the low-luminosity HBLs (e.g., PKS 2155-304: [5]). The foreseen CTA sensitivity (e.g., array E[14]) would allow to densely sample the spectral variability behaviour at the high energy part of the proton synchrotron component. By increasing the target photon density the probability of photomeson production and reprocessing increases. The resulting high energy SED appears smoother, often accompagnied with a higher level of X-ray/soft gamma-ray emission. Because of the higher photomeson production losses the injected proton spectrum is expected to cut off earlier, as does the corresponding gamma-ray spectrum, in comparison to the case of very low photon densities. Examples may be the IBL 3C 66A where the high energy radiation is found to be dominated by a heavily reprocesses proton synchrotron component [29], and the LBL BL Lacertae where pion production losses are found to dominate over proton synchrotron losses with a resulting dominance of strongly reprocessed $\mu / \pi$-synchrotron radiation at X- to gamma-rays [11]. CTA will allow to probe the precise cutoff energy and its possible relation to the AGN subclass of a large sample with high confidence. In the case of FSRQs additional external target photon fields may play a role in forming the overall gamma-ray spectrum also in hadronic emission models, although satisfactorily data representations by hadronic emission models with only internal target photon fields may be found as well (e.g., [12] where modellings of the 2006 flare of 3C 279 have been presented). The large observed luminosity of FSRQs require then large jet powers in the framework of hadronic emission models (see Sect. 3). Because of the cascading of power from high to lower energies, a characteristic feature of hadronic emission models, gamma-ray spectra can naturally be extended to very high energies even with unusually hard spectral shapes (in the galaxy frame) also for FSRQs. Future observations with CTA will be able to verify such behaviour.

\section{References}

[1] Abbasi R et al, (The IceCube collaboration) 2011 Preprint arXiv:1104.5187

[2] Abdo A A et al (The Fermi-LAT collaboration) 2010 ApJ 722520

[3] Abdo A A et al 2011 (The Fermi-LAT collaboration) ApJ in preparation

[4] Aharonian F A 2000 New Astr. 5377

[5] Aharonian F A et al (HESS collaboration), 2005, A\&A 430865

[6] Aharonian F A et al (HESS-collaboration) 2007 ApJ Lett 664 L71

\footnotetext{
${ }^{6}$ Synchrotron radiation from secondary electrons may in some cases also contribute to the low energy hump in the SED .
} 
[7] Aharonian F A and Taylor A M 2010 APh 34258

[8] Albert J et al (MAGIC-collaboration) 2007 ApJ 669862

[9] Atoyan A M and Dermer C D 2003 ApJ 58679

[10] Bednarek W \& Protheroe R J 1999 MNRAS 302373

[11] Böttcher M \& Reimer A 2004 ApJ 609576

[12] Böttcher M, Reimer A, \& Marscher A 2009 ApJ 7031168

[13] Celotti A and Ghisellini G 2008 MNRAS 385283

[14] The CTA Consortium 2010 Preprint arXiv:1008.3703

[15] Dermer C D, Razzaque S, Finke J D, Atoyan A 2009 New Journal of Physics 11065016

[16] Eichler D \& Wiita P J 1978 Nature 27438

[17] Hillas A M 1984 Ann.Rev. A\&A 22425

[18] Levinson A 2000 PhRvL 85912

[19] Mannheim K and Biermann, P L 1992 A\&A 25321

[20] Medvedev M V 2000 ApJ 540704

[21] Mücke A, Rachen J P, Engel R, et al. 2000 NuPhS 80 08/10

[22] Mücke A \& Protheroe R J 2000 AIPC 515149

[23] Mücke A, Engel R, Rachen J P, et al. 2000 CoPhC 124290

[24] Mücke A and Protheroe R J 2001 APh 15121

[25] Mücke A, Protheroe R J, Engel R 2003 et al., APh 18593

[26] Protheroe R J 1997 IAU Coll. 163 Vol. 121585

[27] Protheroe R J \& Mücke A 2001 ASP Conference Proceedings, 250, 113

[28] Rachen J P and Meszaros P 1998 Phys Rev D 58123005

[29] Reimer A, Joshi M, Böttcher M 2008 AIP 1085502

[30] Reimer A 2009 Int. Journal of Modern Physics D 181511

[31] Reimer A and Böttcher 2012, in: “Relativistic Jets from Active Galactic Nuclei” ed M Böttcher, D Harris, H Krawczynski (John Wiley \& Sons) chapter 3

[32] Reimer A 2011a, JCPS, submitted

[33] Reimer A et al 2011b, in preparation

[34] Sikora M and Madejski G M 2000 ApJ 534109 